\title{
On modelling approaches to the influence of mechanical deformation on the micromagnetic activity in a mild steel
}

\author{
A. F. Altzoumailis", V.N. Kytopoulos \\ ${ }^{a *}$ School of Chemical Engineering, National Technical University of Athens, 5 Heroes of Polytechnion \\ Avenue, 15773 Athens, Greece. \\ Email: ajumaily@central.ntua.gr \\ ${ }^{\mathrm{b}}$ School of Applied Mathematical and Physical Sciences, National Technical University of Athens, 5 Heroes \\ of Polytechnion Avenue, 15773 Athens, Greece. \\ Email: victor@central.ntua.gr \\ *Corresponding Author
}

Received: June 10, 2021. Revised: November 3, 2021. Accepted: November 28, 2021. Published: December 20, 2021.

\begin{abstract}
In this study an attempt is made to develop a theoretical modelling by which the influence of certain mechanical deformation factors on the micromagnetic emission behavior of a low-carbon steel can reasonably be described and estimated. This modelling consists of a simple kinetics - kinematics aided approach of the pinning state - controlled domain wall motion by which appropriate specific parameters are introduced. In this aspect the basic notion of specific micromagnetic activity (s.m.a.) is introduced by which the energetic strength of the activity is reflected. In this way, the synergetic effect of the quantitative (count rate) and qualitative (voltage) the detected micromagnetic Barkhausen emission (MBE) is taken into consideration. Thus it is possible, theoretically, to give a prediction of the general trend of changes in the s.m.a. under the influence of the tensile elastic as well as plastic deformation. For instance, one can demonstrate that tensile elastic deformation cannot influence the s.m.a. whereas plastic one leads to an increase in this. Furthermore, one can also predict that increasing permanent (residual) plastic deformation, obtained after unloading from prior tensile loading, leads to an obvious decrease in the s.m.a. Similar decrease in the s.m.a. can also be predicted for increasing rolling deformation by means of the same modelling approach used for the permanent tensile plastic deformation. Owing to the good agreement with the experimental results and the simplicity of the proposed theoretical approaches that can be seen as a promising valuable tool for further similar studies.
\end{abstract}

Keywords: approach, rate of change, s.m.a., pinning strength, pinning spacing, domain walls, deformation.

\section{INTRODUCTION}

Micromagnetic activity in a ferromagnetic material is an intrinsic and characteristic process which can be triggered when a magnetic field and/or stress is applied on the material [1-3]. This activity can be investigated macroscopically by means of various Barkhausen noise emission techniques [4-6]. This noise in turn, is determined by discontinuous (abrupt) changes in the magnetization caused by the applied time-varying magnetic field. The most changes are due to the jumplike-displacements of magnetic domains taking place between internal obstacles, called pinning sites, which are in form of various crystal defects such as stress gradients, grain boundaries, inclusions, dislocation arrays. The jump motion activity depends on the magnetostriction-coupled magnetoelastic interaction between walls and sites. As such the micromagnetic activity can be strongly influenced by internal stress gradient fields caused by applied stress and/or residual stresses. The so arising coupling condition of the magnetic and mechanical properties can be utilized to reveal and analyze certain basic materials parameters concerning the internal stress state, microstructure and grain size $[3,7]$. Theoretical modelling approaches made in this direction by means of Barkhausen noise analysis methods have found in the past relative little application in the practice due to their high complexity [8-15]. The complexity arises mainly from the fact that the Barkhausen noise response of micromagnetic activity presents a strong statistical nature.

This means that the related models contain large stochastic components which lead to many ambiguities concerning the needed statistical approaches. For instance, advanced models of magnetic Barkhausen 
noise emission are based on domain wall dynamics theory by which an attempt is made to describe and explain a very complex physical phenomenon in givenextensive mathematical expressions [16, 17]. Furthermore, this phenomenon is determined by domain wall translation, bending and rotations processes that are affected by highly localized potential fluctuations of stochastic nature. As such, the related multiparametric approaches have prevented these models from a direct introduction into practical-technological applications.

Many applications of Barkhausen noise emission analysis focuses on the evaluation of internal stresses in steel-structured components [18-21]. In these applications, the noise emission is determined by magnetic flux changes which are measured by means of induced-voltage signals obtained from a respective capturing set-up [5]. These signals are conveniently processed to an average root-mean-square (rms) voltage, i.e. $V_{r m s}$, which is a widely used and in general accepted, parameter for the microstructural characterization of materials [19]. Nevertheless, the use of this parameter, individually, implies some experimental difficulties and restrictions concerning critical optimum magnetizing parameters [5]. These difficulties, in turn, may lead to certain ambiguities in data interpretation.

Thereafter, in the frame of these applications it would be valuable at least from academic point of view, to tray to minimize these restrictions and present a much more simple, and "direct" way of theoretical approach by which a prediction of the behavior of the emitted Barkhausen noise under the influence of a given state of deformation can be made. As such, the subject of this study is to propose a heuristic modelling approach in this direction where by using simple mathematical expressions and assumptions the general trend of the micromagnetic activity, reflected by its Barkhausen noise emission response, under the influence of a givenknown state of applied stress-coupled microstructural changes, may reasonably be described and evaluated.

\section{THEORETICAL CONSIDERATIONS}

\section{A. General Presumptions}

For a detection set-up of MBE, the well-known Faraday induction law can generally be set simply as:

$V_{t}=m \frac{d B}{d t}$

where $V_{t}$ is the related voltage induced in the electronic circuit at instant time " $\mathrm{t}$ ", $\mathrm{m}$, a magnetic circuit cuppling constant and B the associated magnetic induction. Thus, the effective Barkhausen Voltage for one individual pulse can be given as:
$V_{e f}=\left[\int_{t_{0}}^{t} V_{t} d t\right]: \Delta t=m\left[\int_{t_{0}}^{t} \frac{d B}{d t} \cdot d t\right]: \Delta t \approx$
$m \Delta B: \Delta t, \Delta t=t-t_{0}=t_{e f}=$
effective pulse duration time.

Thereafter, the measured root-mean-square voltage of the electronically processed pulse signal can be given by:

$\hat{V}_{r m s}=\beta V_{e f}=\beta m \frac{\Delta B}{\Delta t}=m \beta \frac{B_{e f}}{t_{e f}}$

where $B_{\text {ef }}$ is the effective magnetic induction produced during a pulse and ' $\beta$ ' an electronic processing accommodation constant.

Furthermore, one can assume, in a 'zeroth' approximation, an one-dimensional-controlled change in the volume of the planar magnetic domains and as such one can set:

$\hat{B}_{e f} \approx b \cdot l_{p} \propto B_{e f}$

where $\hat{B}_{\text {ef }}$ is the effective magnetic volume swept out by a moving domain wall during duration time, tp, of a jump between two pinning site (obstacle) located at pinning spacing $\mathrm{lp}$, and ' $\mathrm{b}$ ' a domain spacing proportionality accommodation constant . A valuable technique for the analysis of micromagnetic Barkhausen emission (noise) is the so-called pulse - Reight distribution (PHD) [3, 4]. By this technique one can, among others, deduce that groups of magnetic domains, $n_{i}$ may move at an instant time, $t_{i}$, with the associated same average group velocity, $\hat{v}_{i}$, under the influence of a magnetization field. On the other hand, depending on their orientation, several magnetic (block) walls may more simultaneously having different proper velocities. Furthermore, the intensity of Barkhausen noise (energy), signal depends mainly on the rate of magnetic volume change determined by the instant velocity of the moving wall and also by the duration time, $t_{p}$ of the wall jump taking place between pinning sites [4]. In this connection the intensity of the micromagnetic Barkhausen emission measured as (total) $V_{r m s}$ Voltage reflects the jumpsum energy of all domain walls, $\mathrm{N}$, moving in a given time instant of magnetization cycles. Thus the analysis of micromagnetic activity by means of these two signal parameters individually, would obscure many "hidden" details of domain wall motion behavior and lead to ambiguities of data interpretation. Therefore, it is advisable to tray to utilize some appropriate parameters to have a better approach to these details. In this aspect we introduce the first basic relationship as given by:

$\frac{\sum_{i} \hat{V}_{i} n_{i}}{\sum_{i} n_{i}}=\frac{V_{r m s}}{N}=\tilde{J}$

where $\tilde{J}$ is now a specific energetic parameter which better reflects the energetic strength or intensity of the micromagnetic activity. Thereafter, by this parameter one should, formally, introduce the following related relationships which will be used latter:

$\frac{\sum_{i} \tilde{B}_{i} \cdot n_{i}}{\sum n_{i}}=\frac{B_{t o t}}{N}=\tilde{B}$
$\frac{\sum_{i} a_{i} \cdot n_{i}}{\sum_{i} n_{i}}=\widetilde{a}$ 
$\frac{\sum_{i} l_{i} \cdot n_{i}}{\sum_{i} n_{i}}=\tilde{l}$
$\frac{\sum_{i} \cdot n_{i}}{\sum_{i} n_{i}}=\tilde{t}$

where $\tilde{B} \tilde{a}, \tilde{l}$ and $\tilde{t}$ are now the specific parameters of magnetic volume, domain wall acceleration, pinning length (spacing) and jump duration time respectively. It is noted the all of the above presented relationships of specific parameters are substantiated in a first instance by the earlier given interpretation of the PHD- technique as well as the "duality" character of mictomagnetic activity consisting of its mutual qualitative ( voltage ) and quantitative ( number of counts) response . Furthermore, the measured pulse voltage can roughly be given by:

$\hat{V}_{r m s} \cong(\beta \cdot b \cdot m) \frac{l_{p}}{t_{p}} \propto \hat{\theta}_{p}$

Here, $\hat{v}_{p}$ is the average domain wall velocity between given pinning sites (the subscript ' $p$ ' indicates the pinning state which will be disused latter.). At this place it is mentioned that the use of the above introduced basic specific parameter of Eq. (5), seen as a combind expression of jumpsum energy and number of jumps (counts), from the good agreement of presented theory and experimental findings of Fig. (3) one can argue that any changes in the measured count rate of Barkhausen signal might be correlated with respective changes in the number of domain walls overcoming in a given time a fixed number of obstacles $d$ minimize the earlier mentioned experimental ambiguities, restritions and precautions, [5], and as such may give a better approach to a correct interpretation and explanation of the related phenomena. By the way, it is noted that many specific parameters of physics such as specific heat, specific resistance, specific gravity or density specific, strength, may provide a better approach to the related physical and mechanical properties of the materials. Owing to the above - mentioned and physical importance of the specific parameter ' $j$ ' it is also mentioned that its dimension can be approximated as energy per count ( jump event ), giving so an average strength or intensity of domain wall jump event.

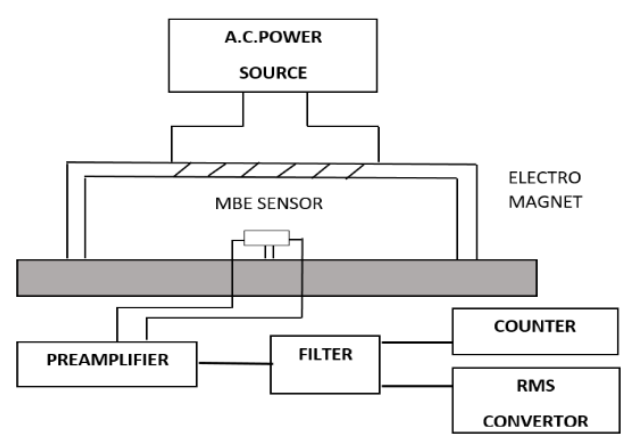

Figure 1: Block diagram of Micromagnetic Barkhausen Emission setup

\section{B. Kinetics}

In the sketch of Fig(2) the static equilibrium state of a domain wall, pinned at two sites (obstacles) by pinning forces $F_{p 1}$ and $F_{p 2}$, under the influence of the effective driving force, $\mathrm{F}_{\mathrm{H}}$, caused by the externally applied magnetization field, $\mathrm{H}_{\mathrm{O}}$, is presented. Under this influence the corresponding back bending or restoring force, $F_{b}$, arises. This is attached at the half pinning distance $1_{p} / 2$, collinear to the driving force. Under these condition the static force equilibrium at a given time $\mathrm{t}_{0}=0$ can be set as follows:

$\vec{F}_{H}-\vec{F}_{b}-2 \vec{F}_{p}=0$

and

$F_{H}=q \cdot H_{0} \sin \varphi$

where $\mathrm{q}$ is a magnetic force accommodation constant and for simplicity $\mathrm{F}_{\mathrm{p} 1}=\mathrm{F}_{\mathrm{p} 2}=\mathrm{F}_{\mathrm{P} \text {. was assumed. }}$.

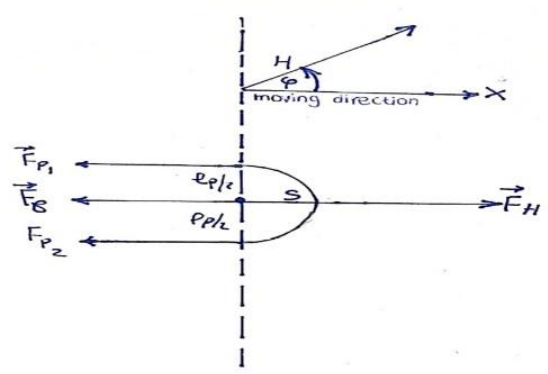

Fig.2: Sketch of static force equilibrium for a given pinning state of a magnetic domain wall.

Furthermore, one can put $F_{b} \propto \frac{\gamma_{w}}{r} \wedge$ forr $\gg$ $s$, resultss $\approx \frac{l_{p}^{2}}{8 r}$, where $\mathrm{r}$ is radius of wall curvature, $\mathrm{s}$, the bowing step, lp the pinning spacing, and $\gamma_{w}$ the specific wall surface energy [22]. Thereafter one can obtain: $F_{b}=8 s \gamma_{w} / l \frac{2}{p}=\eta / l \frac{2}{p}$, where $\eta$ is the specific bowing energy, taken as a accommodation constant in which the bowing step, s, is was assumed to change not appreciable, compared with $l_{p}{ }^{2}$. Related to this and for the sake of completeness concerning the $\gamma_{w}$ - behavior, one should mention that applied stress will cause changes in the internal stress - strain field surrounding the dislocations and hence would induce local fluctuations of interaction between domain walls and dislocations [4]. Such fluctuations would, in turn, cause changes in the specific wall energy $\gamma_{w}$. Such changes would be reflected on a micro - response scale of micromagnetic activity. However, there is a valid reason to accept that the macroscopically measured micromagnetic emission should be scaled on a macro - response determined by the local averages of the magnetoelastic stress - strain fields. Thus, the local fluctuations are often believed to have a limited influence on the salient and reproducible features of the macroscopic behavior. On assuming that local disturbances are a second order effect the problem reduces to the determination of the constitutive laws relating the averages of these fields. In other words, the underlying premise is that the local interaction between dislocations and domain wall weakly depends on the surrounding defects which can, therefore, be smeared out 
into an effective magnetoelastic medium. From the above - mentioned and due to the fact that the intrinsic magnetocrystalline anisotropy dominates over the stress - induced magnetic anisotropy [3], it is envisaged to accept that, $\gamma_{w}$, does not change appreciable and as such cannot influence the pinning state.

Now, for a critical time $t^{*}{ }_{c r}=0^{+}$, it is assumed that the domain wall is unpinned (detached), set free, from the two pinning sites and begins to move under the influence of critical applied force ${F_{H}}^{*}=F^{*}{ }_{b}+2 F^{*}{ }_{p}$ in the direction of $\mathrm{x}$-axis. At this critical instant time, according to the d'Alembert law, the dynamic force equilibrium can be given as:

$$
\begin{aligned}
& \vec{F}_{H}{ }_{H}+\vec{F}^{*}=0 \\
& \text { and } \\
& \vec{F}^{*}=-m \vec{a} \text {, }
\end{aligned}
$$

where $F^{*}$,is the inertial force, ' $a$ ' the corresponding start acceleration of the unpinned domain wall and $\mathrm{m}$ its associated (constant) inertial mass. Taking into consideration all the above relationships one may finally easy abstain:

$a=\frac{2 F^{*}{ }+F^{*}{ }_{b}}{m}=\left[2 F^{*}{ }_{p}+\eta \frac{\gamma_{w}}{l_{p}{ }^{2}}\right]: m$ (15)

This basic equation describes the correlation of the start (break-away) wall acceleration with the pinning state of the critical pinning forces $\left(F_{p}\right.$ and actual pinning spacing $\left(l_{p}\right.$. This equation means that an increase in pinning forces and a decrease in the pinning spacing results in an associated increase in the start acceleration of magnetic wall. Taking into consideration the discussions regarding the specific parameters given by Eqs. (6-9) we will henceforth use the variable of the specific start acceleration denoted by $a_{p}$. As such the basic kinetic Eq. (15) formally becomes a specific one:

$\alpha_{p}=\left[2 \tilde{F}_{p}^{*}+\tilde{\eta} \frac{\tilde{\gamma}_{w}}{\tilde{l}_{p}^{2}}\right]: \widetilde{m}$

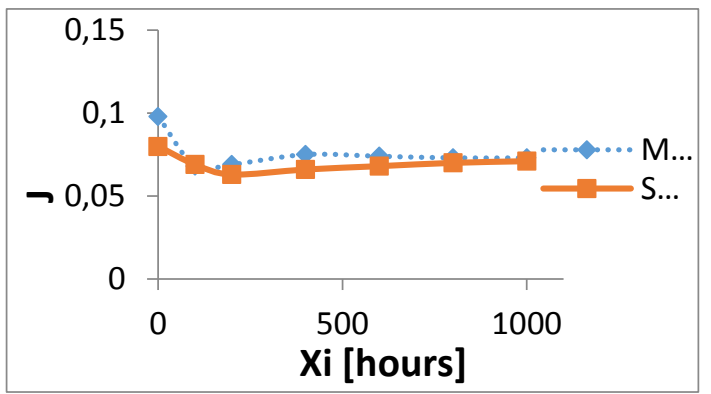

Fig.3: Evaluated s.m.a. (J) for a tensile loading process in function of applied stress.

Where in turn, each variable becomes a specific one. One can see in this formula the interesting (strong) competing effect of critical pinning forces and actual pinning spacing (density) in increasing the start acceleration, fact which will be discussed in the following text and the related appendixes.

Ones detached (unpinned) from its pinning sites the planar domain wall is assumed to move with a constant specific start acceleration during the specific jump duration time $t_{p}$. In this sense the well-known simple kinematic formulas are valid:

$$
\begin{aligned}
& \tilde{b} \tilde{B}_{p} \cong \tilde{l}_{p}=\tilde{a}_{p} \tilde{t}_{p}{ }^{2} / 2 \\
& \tilde{v}_{p}=\tilde{a}_{p} \cdot \tilde{t}_{p}
\end{aligned}
$$

For an incremental change of these variables one can get from Eq. (17)

$\tilde{b} \Delta \tilde{B} \cong \Delta \tilde{l}_{p}=\Delta \tilde{a}_{p} t^{2} / 2+\tilde{a}_{p} \tilde{t}_{p} \Delta t_{p}(19)$

or further

$\frac{\tilde{b} \Delta \tilde{B}}{\tilde{l}_{p}} \cong \frac{\Delta \tilde{l}_{p}}{\tilde{l}_{p}}=\frac{\Delta \tilde{a}_{p} t^{2}}{2 \tilde{l}_{p}}+\frac{\tilde{a}_{p} \tilde{t}_{p} \Delta t_{p}}{\tilde{l}_{p}}$

Thus using Eq. (17) one obtains:

$R_{\widetilde{\mathrm{B}}_{p}} \approx R_{\tilde{l}_{p}}=R_{\tilde{a}_{p}}+2 R_{\tilde{t}_{p}}$

where: $R_{\widetilde{\mathrm{B}}_{p}}=\frac{\Delta \widetilde{B}}{\widetilde{B}_{p}}, R_{\tilde{a}_{p}}=\frac{\Delta \widetilde{a}_{p}}{\tilde{a}_{p}}, R_{\tilde{t}_{p}}=\frac{\Delta \tilde{t}_{p}}{\tilde{t}_{p}}$

denote the corresponding rate of change of these variables. These rates, in turn, are a function of the pinning state determined through the basic equations (11) and (16) by pinning forces and pinning spacing (density) and denoted by the bottom index ' $p$ '.

\section{Elastic deformation}

Within the elastic range of loading the following relationships can be assumed to have an approximated validity [23]:

$d \propto \frac{1}{\sqrt{\sigma}}$
$E \gamma \propto \sqrt{\sigma}$

where $\mathrm{d}$ is the domain spacing(width), othe applied stress and $E \gamma$ the specific surface energy of domain wall. Combining these relationships one obtains: $d \cdot E \gamma \approx$ constant

From above relationships one can deduce that domain spacing decrease whereas specific wall energy increases with applied stress. In this sense such decrease in the domain spacing has been experimentally evidentiated in early studies [23]. Therefore, one may assume that such a decrease in the domain spacing may be correlated with a proportional increase in the number, $N_{w}$, of domain walls fact which would mean:

$d \cdot N_{w} \approx$ constant

Applied stress and magnetizing field, $\mathrm{H}$, have a competing - stimulating effect on the wall motion. Thus, moving walls are temporarily pinned at obstacles determined by internal crystal defects and subsequently unpinned to move (jump) free up to the next obstacle. One can further postulate that the macroscopically measured number (rate) of pulses (counts) could be correlated with the number of pinning/unpinned events determined by the number of domain walls overcoming a given number of obstacles in a given time. This fact suggests to introduce a specific-virtual pinning spacing, $\tilde{l}_{p}^{*}$, given as:

$\tilde{l}_{p}^{*} \propto d \propto \frac{1}{N_{w}} \cdot \frac{1}{\sqrt{N_{p}}}$

where $N_{p} \approx$ constant and $N_{p}$ is the corresponding, fixed, density of "pre-existing" - intrinsic pinning sites in form of grain boundaries, inclusions, precipitates and 
other crystal defects. (For more related details see section (2.5) of this study.)

Furthermore, we assume a magnetoelastic pinning interaction process as given by the differential equation:

$\frac{d E \gamma}{d_{p^{*}}} \propto-F_{p^{*}}$

where $E \gamma$ is the equivalent to the interaction potential wall, $p^{*}$ the effective pinning interactive length and $-F_{p^{*}}$ the related attractive pinning force with $\left|-F_{p^{*}}\right|=$ $F_{p}=$ repulsive - unpinning force .

Consequently, by solving this equation, one may assume the approximated specific expression:

$\tilde{E} \gamma \approx \tilde{\mathrm{p}}^{*} \tilde{F}_{p} \propto \tilde{a}_{p}$

Thereafter combining Eqs (22, 23, 26 and 28) one can easy obtain the basic relationship:

$\tilde{l}_{p}^{*} \cdot \tilde{a}_{p} \approx$ constant

Now from this relationship, in a similar way concerning the procedure of incremental changes in Eq. (17), one can proceed to obtain the following expression:

$$
\begin{aligned}
& \frac{\Delta l_{p^{*}+l_{p^{*}} \Delta a_{p}}}{\tilde{l}_{p}{ }^{*} \tilde{a}_{p}}=0 \\
& \text { and thereafter } \\
& R_{\tilde{l}_{p}{ }^{*}+R_{\tilde{a}_{p}}=0} \\
& \text { where } R_{\tilde{l}_{p}{ }^{*}}=\frac{\Delta_{\tilde{l}_{p}}{ }^{*}}{\tilde{l}_{p}{ }^{*}}, R_{\tilde{a}_{p}}=\frac{\Delta \widetilde{a}_{p}}{\tilde{a}_{p}}
\end{aligned}
$$

are the associated rate of change of specific virtual pinning spacing and start acceleration respectively. Thus, due to Eqs. (22) and (26) the virtual pinning spacing decreases with applied stress resulting in a respective negative rate of change in Eq.

(31) .Consequently from relationships (31) results that:

$$
R_{\tilde{l}_{p}}{ }=-\left|R_{\tilde{l}_{p}}\right|=-R_{\tilde{a}_{p}}
$$

This means that during elastic loading the specific start acceleration of domain walls should increase with elastic stress. In that case from Eq. (21) one can obtain: $R_{t_{p}} \vee \rightarrow-R_{\tilde{a}_{p}} \rightarrow \vee R_{\tilde{l}_{p}} * \mathrm{~V}$

This means that duration time decreases with the same rate as wall acceleration increases. Due to this counterbalance and by virtue of kinematic Eq. (18), results that the specific wall velocity should remain almost constant and as such the measured s.m.a. It should be noted that the Eq. (29) and (32) can also be approached through kinetic Eq .(16) and (28)where , by assuming a negligible change in the specife wall energy and hence the " elastic" pinning torces compared with changes in the dislocation-caused wall pinning forces, an acceleration large arises which is controlled by the pinning spacing [ see Appendix (2)].

\section{Plastic Deformation}

Plastic deformation is characterized by generation of dislocations and formation of dislocation arrays in form of pile-ups and/or tangles as well as cell-structured networks. It becomes obviously that with increasing plastic deformation the dislocation density increases by introducing new pinning sites for moving domain wall. In this sense one could assume that any change in the pinning density, $N_{p}$, should roughly be proportional to the change in the dislocation density $N_{d}$. Thus, it is known that $\sqrt{N_{d}} \propto \frac{1}{\tilde{l}_{p}}$ where $l_{d}$ is the average dislocation spacing [24]. One may also assume $\tilde{l}_{p} \propto \tilde{l}_{p}$ and consequently $\tilde{l}_{p} \propto \frac{1}{\sqrt{N_{p}}} \propto \frac{1}{\sqrt{N_{d}}}$. This formula describes the mode of reduction of pinning spacing with plastic deformation and substantiates the Eq. (26).

From the above mentioned, one can postulate a negative rate of change: $R_{\tilde{l}_{p}}=-\left|R_{\tilde{l}_{p}}\right|$ and also $R_{\widetilde{B}_{p}}=-\left|R_{\widetilde{B}_{p}}\right|$. This means that these parameters should undergo a decremental change i.e. a reduction of their values with plastic deformation. At the same time due to Eq. (33) one may also postulate a positive rate of change in the wall acceleration, i.e. $R_{\tilde{a}_{p}}>0$. Thus, the basic relationship (21) becomes:

$$
2 R_{\tilde{t}_{p}}=-\left[\left|R_{\tilde{l}_{p}}\right|+R_{\tilde{a}_{p}}\right]
$$

Now, by means of this basic formula and demonstrations of Appendix ' $\mathrm{C}$ ' one can establish three variant controlling conditions for approaching the evolution trend of the s.m.a. with plastic deformation. Condition 1: If $\left|R_{\tilde{l}_{p}}\right| \rightarrow \frac{1}{2} R_{\tilde{a}_{p}}$ i.e. the pinning spacing is much more affected than pinning force than from Appendix C2 and basic Eq. (21) we have $R_{\tilde{l}_{p}}=\frac{-3}{4} R_{\tilde{a}_{p}}$. Condition 2: If, pinning strength (force) is much more affected than pinning spacing, than from the Appendix $\mathrm{C} 1$ and basic formula ( 21 ) results: $R_{\tilde{l}_{p}} \rightarrow-\left(R_{\tilde{a}_{p}}\right) \frac{1}{2}$. Condition 3: If both pinning force and spacing are affected with the same degree than from basic Eq. (21) and AppendexC3 we obtain: $R_{\tilde{t}_{p}}=\frac{5}{8} R_{a_{p}}$ It is noted that a fourth variant condition as given by $\left|R_{\tilde{l}_{p}}\right| \gg R_{\tilde{a}_{p}}$ cannot exist since, as shown in appendix ' $\mathrm{C}$ ', the rate of change of acceleration should result by a positive contribution of both pinning force and pinning spacing changes. Thereafter, from the above criteria one may obtain a general relationship in form: $R_{\tilde{t}_{p}}=-\xi R_{\tilde{a}_{p}}$ where $\xi$ is a kinetic analogy constant of pinning state. One can easy observe that for $\xi<1$ an increase whereas for $\xi>1$ a decrease in the measured s.m.a. with plastic deformation should be expected. For $\xi=1$ the s.m.a. should remain almost constant. This observation is based on the simple fact that for decreasing values of $\xi<1$ the increase in the wall acceleration occurs faster than the decrease in the duration time. By kinematic Eq. (18) this fact leads to a net increase in the wall velocity and consequently in the s.m.a. It is mentioned that the obtained values for $\xi=\frac{1}{2}, \frac{5}{8}, \frac{3}{4}$ imposed by the above criteria $1,2,3$, are evaluated by means of Eq. (21) and expressions of sections $\mathrm{C}-1, \mathrm{C}-2, \mathrm{C}-3$ respectively of Appendix C. Thus, one can deduce that the increase in the measured s.m.a. becomes more intensive with a 
decrease of this values. As such, one may further conclude that dominant pinning strength - affected pinning state leads to a more intensive change in the s.m.a. compared to dominant pinning spacing- affected state.

Furthermore, for a given step ' $\mathrm{K}$ ' of plastic deformation the associated kinematic relationships are valid:

$\tilde{l}_{p \kappa}=\tilde{a}_{p \kappa} \cdot \tilde{t}_{p \kappa}{ }^{2}: 2$
$\tilde{V}_{p \kappa}=\tilde{a}_{p \kappa} \cdot \tilde{t}_{p \kappa}$

(the subscript ' $K$ ' denotes the related plastic deformation step). As such, for a following step, $n>K$, of increasing plastic deformation one can postulate:

$\tilde{l}_{p \kappa}>\tilde{l}_{p n} \wedge \tilde{a}_{p \kappa}<\tilde{a}_{p n}$

Combining relationships (35) and (37) one can have:

$\tilde{l}_{p \kappa} / \tilde{l}_{p n}=\frac{\tilde{a}_{p \kappa} \cdot \tilde{t}_{p \kappa}{ }^{2}}{\tilde{a}_{p n} \cdot \tilde{t}_{p n}{ }^{2}}$

and $\frac{\tilde{t}_{p \kappa}}{\tilde{t}_{p n}}>\left(\frac{\tilde{a}_{p n}}{\tilde{a}_{p \kappa}}\right)^{\frac{1}{2}}>1$

Taking into consideration Eqs. (3), (4) and (35), one should now introduce a respective specific voltage parameter which can express a measure of the energetic strength of specific micromagnetic activity (s.m.a.) as follows:

$\tilde{J}_{p \kappa}=m \tilde{B}_{p \kappa} / \tilde{t}_{\kappa}=\frac{m \tilde{l}_{p \kappa}}{\tilde{b} \tilde{t}_{\kappa}}=\frac{m}{b} \tilde{a}_{p \kappa} \cdot \tilde{t}_{\kappa} \cdot \frac{1}{2}(40)$

where ' $\mathrm{m}$ ' is a magnetic accommodation. It results farther:

$\frac{\tilde{J}_{p \kappa}}{\tilde{J}_{p n}}=\frac{\tilde{a}_{p \kappa} \cdot \tilde{t}_{p \kappa}}{\tilde{a}_{p n} \cdot \tilde{t}_{p n}}=\frac{\widetilde{V}_{p \kappa}}{\widetilde{V}_{p n}}$

Combining Eq. (39) and (41) one can find:

$\frac{\tilde{J}_{p n}}{\tilde{J}_{p \kappa}}<\frac{\tilde{t}_{p \kappa}}{\tilde{t}_{p n}}>1$

From the last relationship the following three general variant conditions of s.m.a. may result:

$\frac{\tilde{J}_{p n}}{\tilde{J}_{p \kappa}}>1$
$\frac{\tilde{J}_{p n}}{\tilde{J}_{p \kappa}}=1$

$\frac{\tilde{J}_{p n}}{\tilde{J}_{p \kappa}}<1$

These relationships mean that under certain given conditions the evaluated s.m.a. may increase or remain constant or even decrease with increasing plastic deformation.

\section{Appendix A}

Dislocations accumulated in the same slip plane on an obstacle may form an unstable pile - up array, composed by a number of $\mathrm{n}$ repulsive dislocations whose total energy is $E_{n}>\mathbf{n} . E_{d}$, i.e., higher than the sum of the energies $\mathbf{n}$. $E_{\mathrm{d}}$, of $\mathbf{n}$ individual dislocations [ 24 ]. Since the stress field of a dislocation is a long - range one, it can be assumed that at a critical distances $r$ less than $\mathbf{L}$ of the average slip path, the dislocation pile-up behaves as a super- dislocation with a corresponding superBurges vector $B=n . b,(\mathbf{b}$ is the Burges individual vector). Since the energy of a dislocation is proportional to the square of its Burges vector one can obviously set: $E_{n}=\left(\sum_{i=1}^{n} i b\right)^{2} \gg n b^{2}$

It follows easy that:

$\left(\sum_{i=1}^{n} i\right)^{2}=\left[\frac{n(n+1)}{2}\right]^{2} \gg n$

or in a simpler form:

$\frac{n(n+1)^{2}}{4} \gg 1$

Since, as it can roughly be estimated, the average number of piled - up dislocations is of the order of 5 [24], one can assume an indicative number $\mathrm{n}=5$ and as such from Eq. (c) we have finally: $\frac{180}{4}=45 \gg 1$

This means that the interaction energy of a super dislocation can substantially be about 45-times, higher than that of individual dislocations. Furthermore, in this sense, one could estimate as follows: for the rate of change of formula, (C), one obtains

$\mathrm{R}_{\mathrm{n}}=\Delta \mathrm{n} \quad[1 / \mathrm{n}+\mathrm{N} / \mathrm{n}+1]$ (d)

During plastic deformation the average number of piledlip dislocations may increase between $n=2$ and $n=10$. Now for an incremental step $\Delta \mathrm{n}=1$ one can evaluate from Eq. (d) an average (high) rate of change of about $R_{n}=65 \%$ which would roughly be proportional to the rate of change of the magnetoelastic interaction energy with domain walls and hence in the pinning force $F_{p}$ of kinetic Eq .(16) given in the text.

\section{Appendix B}

On the other hand, under specific metal processing condition, the so-called unlike or attraction type dislocation may be produced [24]. These may form stable arrays of dislocation pile - ups whose effective total, energy, En, this time, is considerable smaller than the sum of the energies $n$. $E_{d}$, of $n$ individual piled - up dislocations. In this case the related effective magnetoelastic energy of interaction with domain walls and hence the pinning forces ' $F_{p}$ ', may considerably be reduced. This in turn, due to Eq. (16), would lead to a respective net decrease in the start acceleration of domain wall. However, under present condition of plastic deformation, the probability of formation of such stable arrays is substantially lower than that of the unstable arrays of like dislocations presented in Appendix A.

\section{RESULTS AND DISCUSSION A. Elastic deformation}

In Fig (3) the behavior of the measured specific magnetic activity (s.m.a.) parameter, $\mathscr{F}$, under condition of applied tensile stress is presented. One can observe that this parameter remain almost constant up to the elastic limit or yield point beyond which an increase in this parameter follows. This behavior, in the elastic range, seems to be consistent with the theoretical predictions 
of modelling approach presented through Eqs. (32) and (33). Detailed phenomenological explanations of the elastic behavior is given by the authors in [25, 26], where that is attributed primarily to the balanced response of applied stress-stimulated increase in the measured Barkhausen energy $\left(\mathrm{V}_{\mathrm{rms}}\right)$ and stress concentration gradients (raisers) - stimulated increase in the pinning strength by which the effective-active number of pinning sites "seen "by the moving domain walls for given path and time range, increased, from the good agreement of presented theory and experimental findings of Fig. (3) One can argue that any changes in the measured count rate of Barkhausen signal might be correlated with respective changes in the number of domain walls overcoming in a given time a fixed number of obstacles in form of internal crystal defects. As such, due to Eqs. (22) and (26) a linear plot of measured count rate versus applied elastic stress, $\sqrt{\sigma}$, could reflex a measure of the crystal defects given by the slope of this linear plot i.e. $\tan \varphi \propto \sqrt{N_{p}}$, where $\varphi$ is the angle between measured signal and stress axis. This technique will be used by the authors in their ongoing related works.

\section{B. Active (on-load) plastic deformation}

On tensile loading beyond yield stress phenomena of dislocation multiplication and creation of substructural defects in form of pile - up groups and tangles as wellas extended cells - structured networks takes place. For instance, the formation of dislocation arrays in iron begins already at tensile strain of about $2 \%$, while at strain $\approx 13-15 \%$ most dislocations are locked into three-dimensional arrays with cells $0,5-1 \mu \mathrm{m}$ in diameter [24].

The development of cell - networks takes place by two opposing process: an increase in the cell wall thickness and a decrease in the interior net surface of cell. The thickness increases by pile - ups and tangles accumulation. As demonstrated in Appendix A, the energy of magnetoelastic interaction of domain walls with formed dislocation pile - ups or tangle arrays may increase considerably with progressive plastic deformation with an average rate as high as $65 \%$. At the same time, the average cell size decreases exponentially towards a limiting level of about $0,5-1 \mu \mathrm{m}$. Therefore, due to these parallel processes a concurrent increase in the pinning forces and a decrease in the pinning spacing takes place. Thus, a more or less equilibrated influence of pinning strength and spacing on the kinetic pinning should be accepted and and the validity of eriterium 3 , expressed by variant condition of formula (43), of an (medium) increase in the measured s.m.a. is substantiated. In fact, this predicted or expected behavior is shown in Fig (3). In this, the experimentally evaluated s.m.a. presents a slight but distinct increase beyond the elastic limit or yield yield-flow stress point of loading. This increase occurs up to the necking or plasticity instability point beyond which, due to elastic unloading phenomena, an decrease follows. This decrease can also be predicted by using the modelling approach to permanent (residual) plastic deformation discussed in section 3.3, and reflected by the general variant condition of Eq. (45) of this study. Nevertheless, at this place it should be pointed out that the desired phenomenological explanations and interpretations concerning the curve of Fig. (3) and other related fintings are given in more details in recent studies of the authors $[25,26]$. These explanations are based on the opposing effects of applied stress and microstructural plastic defects (dislocation volumetric damages) on the magnetoelastic interaction of domain walls with pinning sites. In this sense it is shown that the dominance of applied stress - stimulated increase in the Barkhausen energy (Vrms) over the plastic deformation -caused damage of an increase in the number of pinning sites $(\mathrm{N})$ and strength leads to a net increase in the estimated s.m.a. Beyond the instability point of necking, this dominance is inversed by which a decrease in the s.m.a. is observed.

\section{Plastic unloading}

On unloading, the prior, on - load, active plastic deformation -caused microstructural state is "frozen in" as a reminiscent passive ones. However, in this manner sites of localized residual - permanent stresses are produced. These are related to the earlier mentioned cell - structured dislocation network, created by prior active plastic deformation. This creation as earlier mentioned, is characterized by two opposing effects: an increase in the tangle - structured cell wall thickness and a decrease in the net interior area of cell. On unloading, the interior areas are subjected to compression internal stresses whereas the harder cell walls (nodal sites) to tensile one. Both kinds of these stresses would act as obstacles or pinning sites for moving domain walls. However, due to superposition of these stresses dominant compressive stresses, acting as additional strong pinning sites arise. In this way the specific pinning spacing is further reduced whereas pinning strength is increased compared to the active (on - load) plastic deformation state. This fact leads to a great in the easy domain wall movement and consequently a respective great part of magnetization is forced to occur mostly by reversible wall bowing process taking place between fixed pinning sites. This behavior imposes a change in the Eq. (16) of kinetic pinning state where now $\tilde{a}_{p} \approx 0$ and hence the Eq. (11) of the static equilibrium is valid. It results that, $\frac{\widetilde{\eta}}{\tilde{l}^{2} p}=F_{H}-2 \tilde{F}_{p}$. Furthermore, with a relative good approximation, one might assume a bowing duration time, $\tilde{\mathrm{t}}_{b}$, with a rate of change comparable with that of pinning spacing. Thus, the velocity, $\tilde{v}_{p b}=\tilde{s} / \tilde{t}_{p b}$ is introduced. Therefore, after simplification results: $\tilde{v}_{p b} \propto\left(\tilde{l}_{p}^{2} / \tilde{t}_{p b}\right) \Delta \tilde{F}_{p}$ where $\tilde{V}_{p \beta}$ is the effective specific bowing velocity of the pinned domain walls and $\Delta \tilde{F}_{d}=F_{H}-2 F_{p}$ the effective driving force. In this aspect it is further assumed that the bowing duration time 
may net increase with residual compressive stresses. Since $F_{H}$ and $\tilde{F}_{p}$ are competing forces with upper limiting value, the net driving force $\Delta \tilde{F}_{d}$ would increase with a lower rate as the ratio $\tilde{l}_{p}^{2} / \tilde{t}_{p b}$ decreases with plastic deformation. Therefore, one may argue that $\tilde{v}_{p b}$ in the above equation should undergo a net decrease with plastic deformation causing a respective decrease in the measured s.m.a. In fact, this is reflected in Fig (4), where the evaluated s.m.a., shows a continuous decrease with decreasing specimen thickness or increasing permanent plastic deformation. This behavior is expressed and predicted by the general variant condition of formula 45 . Nevertheless, the formation of a maximization peak observed in Fig (4), at plastic strain about $2 \%$ could reasonably be explained as follows: As mentioned earlier on plastic loading the initiation of cell - structured network sets on at about this strain value during loading process. The absence of residual compression stresses, which otherwise would act as additional strong pinning sites and reduce drastically the effective pinning spacing, leads to a kinetics - controlled pinning state similar to that of prior on - load conditions for which, indifferent which of the presented controlling criteria $1,2,3$ is valid, an increase in the evaluated s.m.a. is expected.

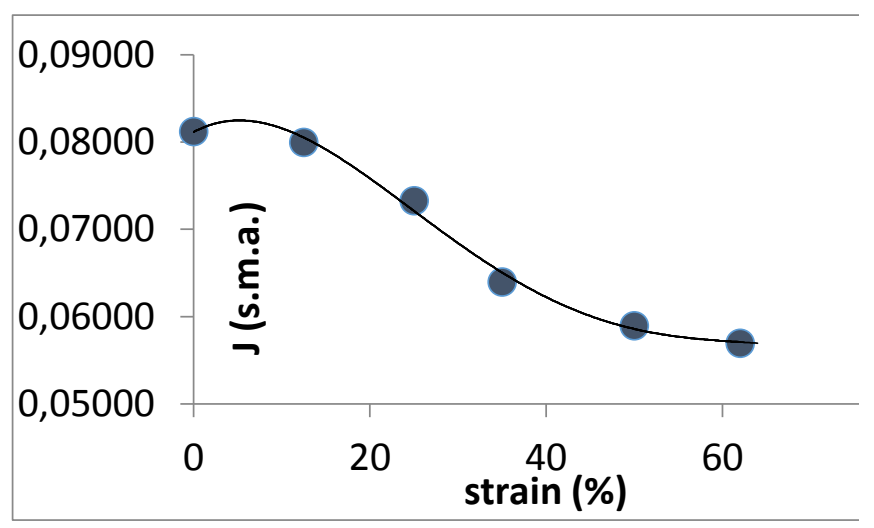

Fig.4: Evaluated s.m.a. (J) after plastic unlocking in function of (permanent) plastic strain.

\section{Appendix "C"}

For an incremental change in the wall acceleration the basic Eq. (16) gives:

$\Delta \tilde{a}_{p}=\left[2 \Delta \tilde{F}_{p}+2 \tilde{\eta}\left|\Delta \tilde{l}_{p}\right| / \tilde{l}_{p}^{3}\right]: m$

(I)

1. If pinning forces are much stronger affected than pinning spacing one may roughly estimate: $R_{\tilde{F}_{p}}=\frac{\Delta \tilde{F}_{p}}{\tilde{F}_{p}} \gg\left|\frac{\Delta \tilde{l}_{p}}{\tilde{l}_{p}}\right|=R_{\tilde{l}_{p}}, \Delta \tilde{F}_{p} \gg \eta\left|\frac{\Delta \tilde{l}_{p}}{\tilde{l}_{p}}\right| \frac{1}{\tilde{l}_{p}{ }^{2}}=\tilde{\eta} R_{\tilde{l}_{p}}$. $\frac{1}{\tilde{l}_{p}^{2}}$ and thereafter

$\Delta \tilde{a}_{p} \approx 2 \Delta \tilde{F}_{p}: \tilde{m}$ and hence $R_{\tilde{a}_{p}} \rightarrow 2 R_{\tilde{F}_{p}}$ and consequently $R_{\tilde{a}_{p}} \gg\left|R_{\tilde{l}_{p}}\right|$
2. If pinning spacing is much stronger affected than pinning strength one may roughly estimate: $\tilde{\eta} R_{\tilde{l}_{p}} \cdot \frac{1}{\tilde{l}_{p}^{2}} \gg \Delta \tilde{F}_{p} \quad, \quad\left|\frac{\Delta \tilde{l}_{p}}{\tilde{l}_{p}}\right| \gg \frac{\Delta \tilde{F}_{p}}{\tilde{F}_{p}} \quad$ and consequently $\Delta \tilde{a}_{p} \approx 2 \tilde{\eta}\left|R_{\tilde{l}_{p}}\right| \cdot \frac{1}{\tilde{l}_{p}^{2} \cdot \tilde{m}}, \quad \tilde{a}_{p} \approx$ $\tilde{\eta} / \tilde{l}_{p}{ }^{2}$ and finally:

$R_{\tilde{a}_{p}}=\frac{\Delta \tilde{a}_{p}}{\tilde{a}_{p}} \rightarrow \frac{2 \widetilde{\eta}\left|R_{\tilde{l}_{p}}\right|}{\tilde{l}_{p}{ }^{2}} / \frac{\widetilde{\eta}}{\tilde{l}_{p}{ }^{2}}=2\left|R_{\tilde{l}_{p}}\right|$

3. If both pinning forces and pinning spacing are affected with same degree than from Eq .( I ) one may obtain:

$\Delta \tilde{a}_{p} \approx \frac{2 \Delta \tilde{F}_{p}}{m} \approx\left(2 \tilde{\eta}\left|\Delta \tilde{l}_{p}\right| / \tilde{l}_{p}^{3}\right): m$ and in a similar way finally $R_{\tilde{a}_{p}} \rightarrow 4\left|R_{\tilde{l}_{p}}\right|$

\section{Rolling deformation}

The complex deformation in rolling can be imagined as superposition of two simpler deformations: tension along the rolling axis and compression perpendicular to the surface of metal. Rolling deformation in polycrystalls involves additional rather complicated factors. Some of these are associated with the fact that the number of types of operative slip systems and respectively the sequences of reorientation may be different owing to different original orientation of grains. For this reason, a multi - component texture is allays existent during deformation in which the number of components begins to diminish considerably on higher deformation. In general, cold rolling deformation should favorise the formation of stable component $\{001\}<110>$, so - called rib texture, which is actually observed in iron. However, from the sequence of complex texture transition this component will appear in iron and other b.c.c. polycrystalls only on heavy deformations. Owing to the strong anisotropic magnetic properties of iron it was found that this texture is an easy magnetization one in which the magnetic flux goes almost parallel to the rolling direction of sheets and would enhance the measured Barkhausen signal. Nevertheless, in sheets of low carbon steel the surface layers differ from deeper one by strong scatter of the easy magnetization direction $\{001\}<110>$ fact which would produce an effective weak component of this orientation and hence a low contribution to the magnetic signal.

In rolling, the zones where the flow of the metal during deformation has been more intensive, such as surface layers, are subjected to dominating compressive residual stresses. In contrast internal layers with lower rate of metal slow experience dominating residual tensile stresses. From the above - mentioned and owing to the fact that micromagnetic Barkhausen emission is related with the first surface - subsurface layers of specimen one may deduce that residual compressive stresses, acting as strong pinning strength sites, have a controlling influence on the detected signal in reducing the related s.m.a. In fact, this is very 
well corroborated by Fig (5), where a continuous decrease in the measured - evaluated s.m.a with reduction of specimen thickness or increasing residual plastic strain occurs. As such, the desired explanations for this behavior are similar with those given earlier for the case of unloading after tensile plastic deformation. It is pointed out that the curve of Fig. (5), was constructed by taking into consideration certain representative rolling deformation measurements given in Eq. [27]. Kindly supplied by the respective author and presented in $\operatorname{Fig}(6)$,veloped to better describe and predict trends of changes in the micromagnetic emission response of a mild - steel under the influence of certain mechanical deformation factors. Thus, by this theoretical approach one can demonstrate that the domain wall motion may be controlled by the associated pinning microstructural state which in turn is determined by the parameters of pinning strength (force) as well as pinning spacing. All parameters of the theoretical modelling were referred to specific one by which the specific energetic notion of the strength of micromagnetic activity can be introduced. This seems to conveniently reflect the synergetic effect of the quantitative (count rate) as well as qualitative (voltage) mutual response of measured micromagnetic emission. Based on the simplicity of the proposed theoretical approach it is believed that an acceptable good agreement of experimental data with theoretical predictions was obtained. In particular, it was possible to give a reasonable prediction of an equilibrated behavior of the measured s.m.a. within the elastic deformation range. On the other hand, theoretical predictions of an increase in the s.m.a. with on - load plastic deformation are also in good agreement with obtained experimental data. Furthermore, one can also show that data obtained from permanent plastic deformation due to unloading after previous plastic loading as well as from rolling procedure are consistent with theoretical predictions of a decrease in the s.m.a. with plastic deformation. The desired explanations of the related phenomena are given on the basis of a domain wall motion controlled by the rate of change in the pinning strength and pinning spacing. This change in turn can be correlated with certain deformation - induced microstructural changes in form of dislocation arrays and other crystal defects. Owing to the simplicity of the proposed approach it is believed that this can be accepted as a valuable tool for further similar investigations.

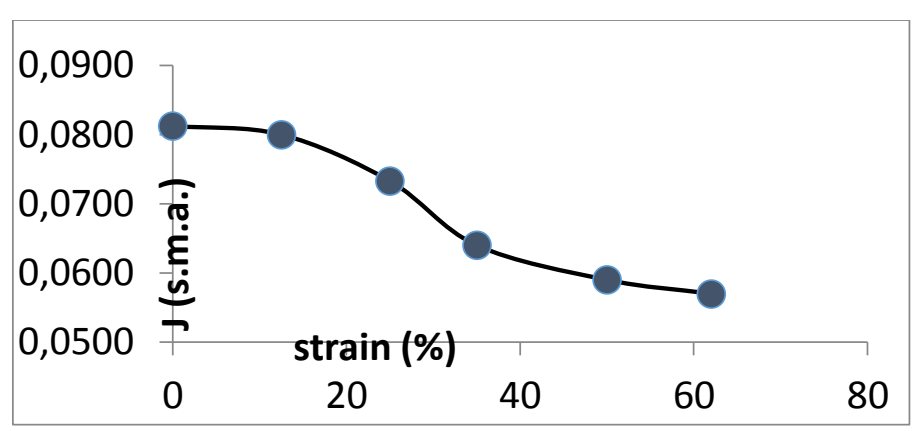

Fig.5: Evaluated s.m.a. (J) for rolling deformation in function of thickness reduction.

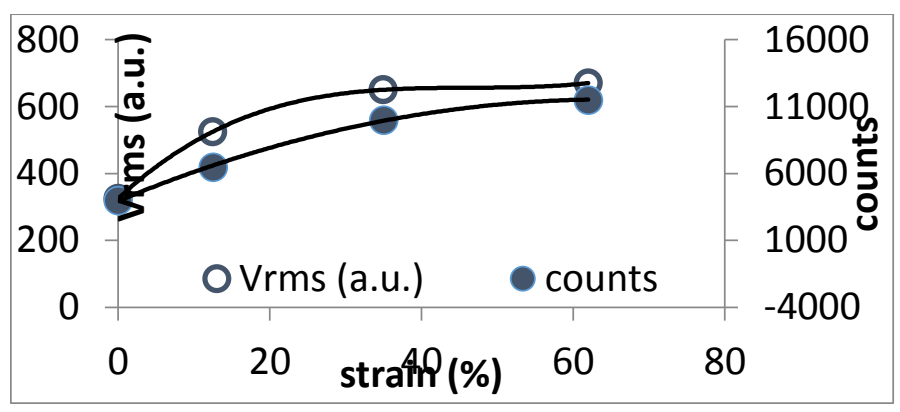

Fig.6: Barkhausen energy and count measurements for rolling deformation in function of thickness reduction, by courtesy of [27].

\section{CONCLUSIONS}

In this study a kinetics - kinematics - aided modelling approach has been developed to better describe and predict trends of changes in the micromagnetic emission response of a mild - steel under the influence of certain mechanical deformation factors. Thus, by this theoretical approach one can demonstrate that the domain wall motion may be controlled by the associated microstructural pinning state which in turn is determined by the parameters of pinning strength (force) as well as pinning spacing density. All parameters of the theoretical modelling were referred to specific one by which the specific energetic notion of the strength of micromagnetic activity can be introduced. This seems to conveniently reflect the synergetic effect of the quantitative (count rate) as well as qualitative (voltage) mutual response of measured micromagnetic emission. Based on the simplicity of the proposed theoretical approach it is believed that an acceptable good agreement of experimental data with theoretical predictions was obtained. In particular, it was possible to give a reasonable prediction of an equilibrated behavior of the measured- evaluated s.m.a. within the elastic deformation range. On the other hand, theoretical predictions of an increase in the s.m.a. with on - load plastic deformation are also in good agreement with obtained experimental data. Furthermore, one can also show that data obtained from residual plastic deformation after prior tensile plastic 
loading as well as from rolling deformation are consistent with theoretical predictions of a decrease in the s.m.a. with plastic deformation. The desired explanations of the related phenomena are given on the basis of a domain wall motion, controlled by the rate of change in the pinning strength and pinning spacing. This change, in turn, can be correlated with certain deformation - induced microstructural changes in form of dislocation arrays and other crystal defects. Owing to the simplicity of the proposed approach it is believed that this can be accepted as a valuable tool for further similar investigations.

\section{REFERENCES}

[1] H. Kronmuller, M. Fahnle, in "Micromagnetism and the Microstructure of Ferromagnetic Solids", Cambridge University Press, Cambridge, 2003.

[2] R. S. Tebble, "The Barkhausen effect", Proc. Phys.Soc., London, B86:1017-1032, (1995).

[3] Thomas W. Krause, L. Clapham, Andreas Pattantyus, David L. Atherton, “' Investigation of stress- depeudut magnetic easy axis in steel using magnetic Barkhausen noise “J. Appl. Phys. , 79 (8), (1996) , pp.4242 - 4252.

[4] L. J. Dijkstra and U.M.Martius, "Domain Pattern in Silicon -Iron under Stress", Reviews of Modern Physics, Val.25, Nr.1, 146-150, (1953).

[5] P. Zerovink, J. Grum,G. Zerovinik," Determination of hardness and Residual-Stress Variations in Hardened surface Layers with Magnetic Barkhausen Noise ", IEEE-Transactions on Magnetics , 46,(3),899-904, (2010).

[6] L. B. Sipahi, DC. Jiles, D. Chandler," Comprehensive analysis of Barkhausen emission spectra using pulse height analysis, frequency spectrum, and pulse wave form analysis", J.Appl.Phys.,73,(10),5623-5625, (1993). [7] V. Moorthy, B.A.Shaw, "Magnetic Barkhausen emission measurements for evaluation of materials properties in Gears", NDT \& INT, 23, 317 - 347, (2008). [8] O. K. ypris , I.C. Nlebedim , D.C. Jiles , “Measuring stress variation with depth using Brkhausen signals “, J. Magn. Materials, 377-395, 407, (2016).

[9] D. C. Jiles, L. B. Sipahi and G. Williams "Modelling of mictomagnetic Barkhausen activity using a stochastic process extension to the theory of hysteresis. J. Appl. Phys., 73, 5830, (1993).

[10] B. Durcharne, M.Q. Le. G. Sebald, P.J. Cottinet, D. Guyomar, Y. Hebrard " "Characterization and modeling of magnetic domain wall dynamics using reinitiated by stresses loaps from Barkhausen noise “, J. of Mag. And Mag. Mat. Pp231-238, (2017).

[11] I. Sablik etal: "Micromagnetic model for biaxial stress effects on magnetic properties", J. Magn. Magn. Mater. 132,: 131-148, (1994).

[12] M.J. Sablik, "A moclel for the Barkhausen noise power as a function of applied magnetic field and stress", J. A.ppl. Phys. , 74,5898- 5900, ( 1993).
[13] Hunt Michael, “Analytical expressions for Barkhausen jump size distnbution, IEEE Trans Magn., 30, 4356-4358, (1994).

[14] C. C. H. Lo, E. Kinser, and D. C. Jiles, "Modelling the interrelating effects of plastic deformation and stress on magnetic properties of materials", Journal of Applied Physics 93, 6626 (2003); https://doi.org/10.1063/1.1557356

[15] O. Kypris, I.C. Nlebedim, D.C. Jiles, "A model for the Barkhausen frequency spedrum as a function of applied stress “, J.Appl. Phys. 115, (8), 083906

[16] B. Alessandro, C. Beatrice, G. Bertotti, A. Montorsi," Domain wall dynamics and Barkhausen effect in metallic ferromagnetic materials", 1.Theory, J. Appl. Phys.-68(6), 29012907, (1990).

[17] Hunt / Michael, "Analytical expressions for Barkhausen jump size distnbution “, IEEE Trans Magn., 30, 4356-4358, (1994).

[18] A.Lasaosa, R. Gurruchaga, V. Garcia Navas, A.Martinez-de-Guerenu"Charactenzation of in - depth stress state by magnetic Barkhausen noise on machined steel acquiring frequency bands", Advanced Mat.Res.Va1996,373-379, (2010).

[19] D.O'Sullivan, M. Conterell, D.A. Tanner, I. Meszaros," Characterization of ferritic stainless steel by Barkhausen technique", NDT \&International, 37, 489496, (2004).

[20] X.Kleber, S.Pirfo Barroso, "Investigation of shot pelned austenitic stainless stell 30L by means of magnetic Barkhausen noise," Mt. Sci. Engn., A, 527, 60466052, (2010).

[21] M. Lindgren, T. Lepisto, "Effect of prestraining on Barkhausen noise VS.Stress relation ", NDT8CE International, 34,337-344, 2001.

[22] S. Chikazumi, "Physics of magnetism “, Wiley, N.Y. (1994).

[23] L. J. Dijkstra and U.M.Martius, "Domain Pattern in Silicon - Iron under Stress", Reviews of Modern Physics, Val.25, Nr. 1, 146-150, (1953).

[24] P. Polukhin , S. Gorelik , V. Vorontsov , “ Physical principles of plastic Deformation “, ( in English ), Mir publishers, MOSKOW , ( 1983) .

[25] A. F. Altzoumailis, V. N. Kytopoulos, "Characterization of a mild steel by its mutual Tensile Mechanical and Micromagnetic emission response after corrosion in $\mathrm{NaCl}$ - water Solution: a combined semiquantitive approach" INTERNATIONAL JOURNAL OF MATERIALS DOI: 10.46300/91018.2021.8.3. Volume 8, 2021

[26] A. F. Altzoumailis, V. N. Kytopoulos, "On Novel Aspects of Hydrogen Effects on Applied Stress Coupled Micromagnetic Activity in a Mild Steel after Exposure to $\mathrm{NaCl}$ - Water Solution: A Combined Approach", INTERNATIONAL JOURNAL OF MATERIALS DOI: 10.46300/91018.2021.8.4. Volume 8,2021

[27] Kosmas, Konstantinos of Nikolaos, "Development of electromagnetic non-destructive methods in magnetic metallic materials", NTUA, DOI 10.12681/eadd/23322, [2009].

\section{Creative Commons Attribution License 4.0} (Attribution 4.0 International, CC BY 4.0)

This article is published under the terms of the Creative Commons Attribution License 4.0

https://creativecommons.org/licenses/by/4.0/deed.en_US 